\title{
Blastomycosis and Pregnancy: An Unusual Postpartum Disease Course
}

\author{
David Surprenant $^{a} \quad$ Monika Kaniszewska $^{a} \quad$ Kelli Hutchens $^{c}$ \\ Christine Go ${ }^{a} \quad$ Paul O'Keefe $^{b} \quad$ James Swan ${ }^{a} \quad$ Rebecca Tung $^{a}$ \\ Divisions of ${ }^{\mathrm{a}}$ Dermatology, ${ }^{\mathrm{b}}$ Infectious Diseases and ${ }^{\mathrm{C}}$ Pathology, Loyola University Medical \\ Center, Maywood, Ill., USA
}

\author{
Key Words \\ Blastomycosis · Cutaneous · Pregnancy
}

\begin{abstract}
Blastomyces dermatitidis is responsible for systemic mycoses. It is predominantly caused by inhalation of spores and often manifests as pneumonia, which can potentially disseminate; however, direct cutaneous inoculation may also occur. Blastomycosis in the perigravid period is exceedingly rare. The partial immunosuppressive state induced by pregnancy can engender more severe infections and is associated with a risk of vertical transmission. Published cases describe postpartum symptomatic improvement accompanying immune reconstitution, even in the absence of treatment. We present a 31-year-old gravid female with multifocal cutaneous blastomycosis. After delivering a healthy full-term infant with no evidence of congenital infection, the patient's cutaneous lesions continued to worsen. At 6 weeks postpartum she was treated with oral itraconazole and demonstrated clinical improvement after 5 months of therapy. This case highlights the importance of prompt disease recognition, understanding of risk factors and initiation of appropriate antifungal therapy of blastomycotic infection occurring in the unique setting of pregnancy.

(C) 2015 S. Karger AG, Basel
\end{abstract}

\section{Introduction}

Blastomyces dermatitidis is a dimorphic fungus responsible for systemic mycoses predominantly caused by inhalation of spores [1]. Infection may present as acute or chronic pneumonia with potential dissemination via hematogenous or lymphatic spread, occasional- 
Surprenant et al.: Blastomycosis and Pregnancy: An Unusual Postpartum Disease Course

ly progressing to fatal disease [2-4]. Overall, skin involvement has been reported in 40-80\% of cases [5]. Even in endemic regions, blastomycosis infection during pregnancy is exceedingly rare. A comprehensive review by Lemos et al. [6] of records at the University of Mississippi Medical Center (located in an endemic region), as well as of published cases, revealed only 19 cases of blastomycosis infections during pregnancy over a span of 108 years.

It has been suggested that pregnancy results in a partial immunosuppressive state, which may predispose gravid women to infection with fungal organisms [6]. Further, infection with a nonobligatory opportunistic fungus, such as Blastomyces, may be more severe in immunosuppressed individuals, making disseminated disease more likely $[4,6,7]$. Therefore, blastomycosis during pregnancy is particularly concerning. Beyond the concern for maternal health, blastomycosis during pregnancy also carries a risk of potentially fatal congenital blastomycosis of the newborn [6].

In published cases of blastomycosis during pregnancy, all mothers showed considerable postpartum regression of disease, even if no treatment was administered. This improvement likely resulted from recovery of their immunocompetent state [6]. Our case represents a rare instance of cutaneous blastomycosis during pregnancy. Further, our patient did not display the expected postpartum regression of blastomycosis symptoms seen in all previously reported cases. Rather, her cutaneous blastomycosis continued to progress beyond delivery of a healthy child.

\section{Case Report}

An otherwise healthy 31-year-old Hispanic female, 37 weeks pregnant, presented with complaint of a rash on her left thigh and right cheek that had been present for over 1 year. The lesions were pruritic, growing in size, friable with intermittent bleeding and were also tender to palpation. The patient denied any recent travel and any affected close contacts. She denied having fever, chills, respiratory symptoms or other cutaneous complaints. A review of systems was unremarkable.

Physical examination revealed two lesions: a $3.0 \times 1.5 \mathrm{~cm}$ pink, firm, oval nodule with hyperpigmented borders which was studded with pustules on the left distal thigh (fig. 1a) and a $3.3 \times 2.0 \mathrm{~cm}$ annular well-demarcated, pink verrucous plaque with surrounding erythema and overlying scale and hemorrhagic crusting on the right cheek (fig. 1b).

Biopsy and tissue cultures were performed. Histological analysis revealed pseudoepitheliomatous hyperplasia with underlying dense superficial and deep mixed cell infiltrate composed of neutrophils, lymphocytes, histiocytes and multinucleated giant cells (fig. 2a). A few scattered round to oval organisms with refractile cell walls in the cytoplasm of giant cells, consistent with B. dermatitidis, highlighted by Grocott-Gomori methenamine silver stain, were identified (fig. 2b). Well-formed necrotizing granulomas and large neutrophilic abscesses, consistent with fungal infection, were readily identifiable (fig. 2c). Fungal culture grew B. dermatitidis, which was confirmed by DNA probe.

While amphotericin B is the drug of choice for blastomycosis in pregnancy due to potential teratogenicity of the azole antifungals [6], our patient opted to defer systemic therapy until after delivery due to the proximity of her due date at the time of diagnosis and her absence of systemic symptoms. She delivered a healthy full-term baby with no evidence of Blastomyces infection.

Postpartum, the patient's facial lesion continued to progress and began draining purulent material at the lateral edge. Evaluation for pulmonary infection with a chest radiograph was negative and the patient continued to lack systemic symptoms. At this point, treatment 
Surprenant et al.: Blastomycosis and Pregnancy: An Unusual Postpartum Disease Course

with itraconazole was discussed; however, the patient was unable to proceed with therapy due to lack of insurance coverage. At approximately 6 weeks postpartum, itraconazole was obtained. Since both the facial and thigh lesions had increased in size during postpartum follow-up, she was started on a dose of $200 \mathrm{mg}$ orally twice daily. After 5 months of therapy, both the facial and thigh lesions demonstrated favorable signs of regression (fig. 3 ).

\section{Discussion}

Blastomycosis during pregnancy is exceedingly rare, with few reported cases over the past century [6]. Given the immunosuppressed state of pregnancy, gravid women are more likely to develop blastomycosis as well as disseminated disease, leading to potential risk of congenital infection in the fetus $[3,6,8]$. We suspect that pregnancy contributed to exacerbation of a preexisting case of cutaneous blastomycosis in our patient. Her decision to forgo treatment until after delivery of her child was warranted due to lack of systemic symptoms. While she experienced a progression of cutaneous blastomycosis during and after pregnancy, she fortunately had no pregnancy-related complications and delivered a healthy infant with no signs of systemic Blastomyces infection.

Published cases of blastomycosis during pregnancy have described postpartum regression even in the absence of treatment [6]. In contrast, our patient demonstrated progression of the current cutaneous blastomycosis at 6 weeks postpartum, yet did not develop additional cutaneous lesions or signs of dissemination to noncutaneous sites. Of the reported cases of blastomycosis in pregnancy, patients with acute respiratory distress had a worse prognosis [6].

The treatment of choice for blastomycosis during pregnancy is lipid formulation amphotericin B, 3-5 mg/kg/day, which is deemed effective and safe [4]. Lipid formulated preparations of amphotericin B are used more commonly, given their greater tolerability and lesser toxicity. Systemic therapy may prevent fetal transmission, although definitive evidence is lacking [4].

Although direct inoculation of B. dermatitidis may occur, most cases of cutaneous disease occur after lymphohematogenous spread from a primary lung infection [8]. Therefore careful evaluation is imperative, especially in the setting of pregnancy, to exclude systemic disease necessitating therapy.

Itraconazole is the drug of choice among available azole antifungal drugs to treat mildto-moderate blastomycosis without central nervous system involvement occurring in nonpregnant individuals. Cure rates of $90 \%$ have been reported after a 6-12-month regimen of 200-400 mg per day [7]. We present a case of progressive cutaneous blastomycosis in a gravid female treated with a 6-month course of itraconazole $200 \mathrm{mg}$ p.o. b.i.d. in the postpartum interval. This patient did not exhibit regression after delivery, as would be expected with immune reconstitution. Instead, she only began to show signs of clinical improvement after initiation of treatment.

This case underscores the importance of disease recognition and prompt appropriate treatment of blastomycosis if systemic involvement is suspected. Treatment, especially in unique circumstances like pregnancy, can halt further progression and may prevent possible transplacental dissemination. Our report describes conservative management of multifocal cutaneous blastomycosis in a gravid female lacking systemic symptoms, emphasizing the need to institute treatment in the postpartum period if cutaneous disease is progressive. 
Surprenant et al.: Blastomycosis and Pregnancy: An Unusual Postpartum Disease Course

\section{Statement of Ethics}

The subject of this case report provided informed consent and the study protocol complied with institutional and IRB guidelines.

\section{Disclosure Statement}

The material presented in this case report represents original work and none of the involved authors have any conflict of interest disclosures to make.

\section{References}

1 Tarr M, Marcinak J, Mongkolrattanothai K, Burns JL, Wheat LJ, Durkin M, Ismail M: Blastomyces antigen detection for monitoring progression of blastomycosis in a pregnant adolescent. Infect Dis Obstet Gynecol 2007;2007:89059.

2 Bonifaz A, Vázquez-González A, Perusquía-Ortiz AM: Endemic systemic mycoses: coccidiomycosis, histoplasmosis, paracoccidioidomycosis and blastomycosis. J Dtsch Dermatol Ges 2011;9:705-715.

3 Pipitone MA, Gloster HM: A case of blastomycosis in pregnancy. J Am Acad Dermatol 2005;53:740-741.

4 Chapman SW, Dismukes WE, Proia LA, Bradsher RW, Pappas PG, Threlkeld MG, Kauffman CA; Infectious Diseases Society of America: Clinical practice guidelines for the management of blastomycosis: 2008 update by the Infectious Diseases Society of America. Clin Infect Dis 2008;46:1801-1812.

5 Ortega-Loayza AG, Nguyen T: Cutaneous blastomycosis: a clue to a systemic disease. An Bras Dermatol 2013;88:287-289.

6 Lemos LB, Soofi M, Amir E: Blastomycosis and pregnancy. Ann Diagn Pathol 2002;6:211-215.

7 Dismukes WE, Bradsher RW Jr, Cloud GC, Kauffman CA, Chapman SW, George RB, Stevens DA, Girard WM, Saag MS, Bowles-Patton C: Itraconazole therapy for blastomycosis and histoplasmosis. NIAID Mycoses Study Group. Am J Med 1992;93:489-497.

8 Motswaledi HM, Monyemangene FM, Maloba BR, Nemutavhanani DL: Blastomycosis: a case report and review of the literature. Int J Dermatol 2012;51:1090-1093.
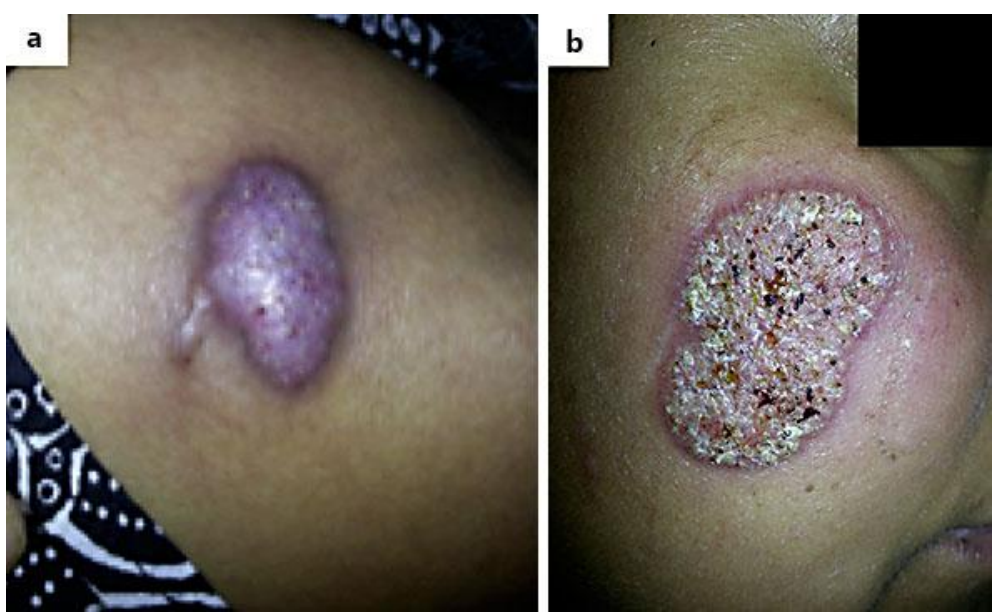

Fig. 1. Initial presentation of cutaneous lesions on the left distal thigh (a) and the right cheek (b). 
Surprenant et al.: Blastomycosis and Pregnancy: An Unusual Postpartum Disease Course
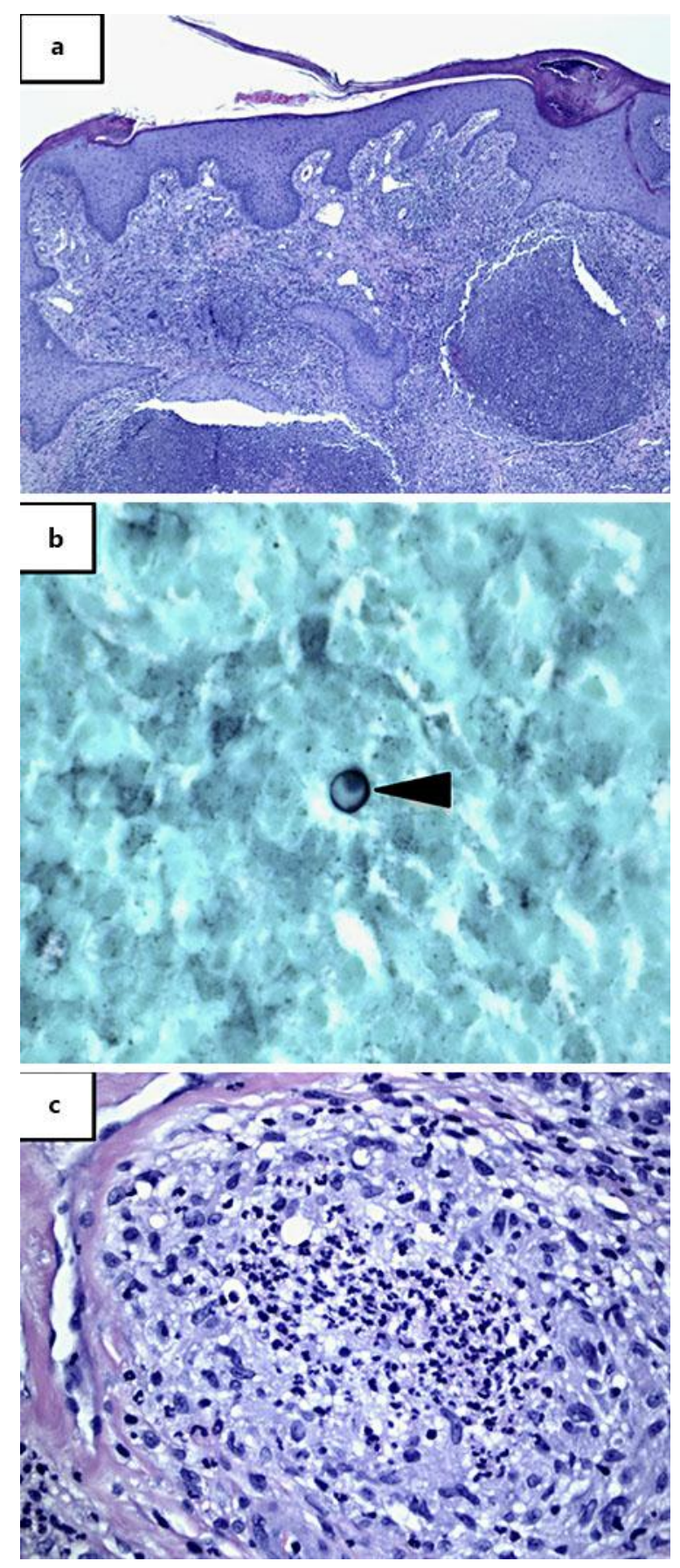

Fig. 2. a, b Pseudoepitheliomatous hyperplasia with underlying abscess formation $(\mathbf{a}, \times 200)$, with rare B. dermatitidis yeast forms highlighted by Grocott-Gomori methenamine silver stain $(\mathbf{b}, \times 600)$. c Focal granuloma formation with central abscess $(\times 400)$. 
Case Reports in
Dermatology

Surprenant et al.: Blastomycosis and Pregnancy: An Unusual Postpartum Disease Course
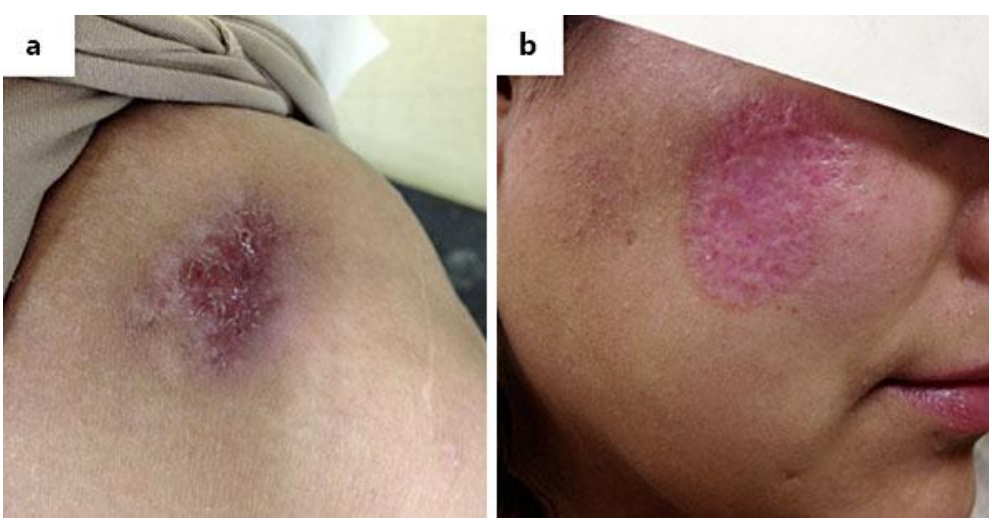

Fig. 3. Lesion on the left distal thigh (a) and the right cheek (b) after 5 months of treatment with itraconazole. 